\title{
How studies on inflamm-aging may help to understand and combat COVID-19 pandemic.
}

Gianluca Storci $^{1 \#}$, Francesca Bonifazi ${ }^{2}$, Paolo Garagnani ${ }^{1,3}$, Fabiola Olivieri ${ }^{4,5}$ and Massimiliano Bonafè $^{1 \#}$

${ }^{1}$ Department of Experimental and Diagnostic Medicine University of Bologna

${ }^{2}$ Hematology department, S. Orsola-Malpighi, Bologna, University Hospital, Bologna, Italy

${ }^{3}$ Clinical Chemistry, Department of Laboratory Medicine, Karolinska Institutet at Huddinge University Hospital, Stockholm, Sweden.

${ }^{4}$ Department of Clinical and Molecular Sciences (DISCLIMO), Università Politecnica delle Marche, Ancona, Italy;

${ }^{5}$ Center of Clinical Pathology and Regenerative Therapy, IRCCS INRCA, Ancona, Italy.

\# equally contribution

Correspondence to: gianluca.storci@unibo.it; gianluca.storci@gmail.com massimiliano.bonafe@unibo.it

\begin{abstract}
More than 1,000,000 confirmed cases of COVID-19 have been registered worldwide since the beginning of the pandemic in Wuhan on December 2019. The high mortality rate of COVID-19 is associated with age, gender and the presence of comorbidities. Biochemical data have shown that COVID-19 patients develop a local and systemic hyper-inflammatory response associated with poor outcome. Therefore, the understanding of the biological mechanisms underlying SARS-CoV-2induced inflammation is a compelling need. Following this reasoning, here we will focus on the importance of the progressive age-related development of a pro-inflammatory state (aka inflammaging) in the understanding of the unbalanced inflammatory response against SARS-CoV-2 in aged people. In particular, we underpin the role of mitochondrial DNA and genomic DNA telomeric sequences in local and systemic mechanisms of inflammation. Indeed, the leakage of mtDNA out of its natural compartment (i.e. the mitochondrion), into the cytoplasm and in the extracellular environment is a powerful trigger of innate immunity and inflammation, as part of an evolutionaryconserved signaling mechanism of cellular damage (e.g. viral infection). High levels of circulating mtDNA are increased in aged people and set up as inflammatory markers of poor prognosis in intensive care unit patients. In turn, telomeric DNA, which can be released into the cytoplasm and in the extracellular environment upon cell damage, has been proven to exert potent antiinflammatory activity. Since that aged people (particularly those affected by co-morbidity) are
\end{abstract}


equipped with shortened telomeres, we posit that, in aged people affected by COVID-19 the release of mtDNA, coupled with insufficient telomeric DNA favors the onset of a detrimental inflammatory response. In this regard, we highlight that the mechanism of action of some currently used drugs, as well as potential new ones may be better understood under the light of the above-depicted theoretical framework thus explaining how studies on inflamm-aging may help to understand and combat COVID-19 pandemic.

Keywords: Covid-19; SARS-CoV-2; Inflamm-aging; mtDNA; Telomere; Inflammation

\section{Introduction}

The ongoing COVID-19 pandemic caused by the SARS-CoV-2 corona virus is causing a global health emergency ${ }^{1}$. The rapid SARS-CoV-2 infectivity and the appearance of serious and fatal respiratory complications in a huge number of patients represent unprecedented threats for all health systems worldwide. The clinical manifestations in the most severe patients are accompanied by an aberrant inflammatory response or "cytokine storm" in which interleukin-6 (IL-6) values stands out among all ${ }^{2,3}$. Thanks to this evidence, patients with SARS-CoV-2 are expected to benefit of administration of the monoclonal antibody against IL-6/IL-6 receptor to taper the cytokine release syndrome (NCT04306705). As expected, a tight relationship among viremia/RNAemia, serum IL-6 levels and poor clinical outcome has been described in COVID-19 patients. Therefore, antiviral and immunosuppressive therapies, alone or in combination, are being undertaken by repurposing a variety of anti-viral/inflammatory drugs ${ }^{4}$. Nevertheless, the extreme virulence of the infection in a substantial proportion of patients (9-11\%), coupled with its impressive infectivity (an estimated infectivity coefficient between 2 to 3 in spite of 1.4 of seasonal flu) and an unprecedented worldwide spread (about 480.000 affected people on March 26 $6^{\text {th }} 2020$; https://www.who.int/docs/default-source/coronaviruse/situation-reports/20200317-sitrep-57-covid19.pdf), underpins the urgent need to understand the pathogenesis and the molecular mechanisms of the most severe manifestation of COVID-19 disease. The data on pandemic show that the pathogenesis and the clinical outcome of SARS-CoV-2 infection are related to the age of the infected patients, irrespective of ethnicity ${ }^{\mathbf{5 , 6}}$. In Italy, at March $24^{\text {th }}$ median age of dead people is 78 years of age, and about $1 \%$ of dead people are less than 50 years of age. Most of them (51.2\%) were affected by at least three age-related diseases (cardiac ischemia, hypertension, type II diabetes mellitus, among others, https://www.epicentro.iss.it/). Mostly, but not exclusively in the elderly, SARS-CoV-2 infection was associated with an uncontrolled systemic sepsis/cytokine release syndrome reaction (https://www.iss.it/asset_publisher. Report n.15). Studies on macaques carried out with SARS-CoV-1 (which was responsible for the 2002-2003 SARS epidemic) show that, 
despite the viral replication rate is similar between young and aged individuals, the lungs of the older ones are affected by an uncontrolled inflammatory response, associated with an extremely deranged histo-pathology ${ }^{7}$. Such a striking age-related trend in COVID-19 pandemic spread recalls mechanisms related to the rate of aging that can be recapitulated by the portmanteau word inflammaging $^{8-10}$. Inflamm-aging depicts the drift of systemic low-grade inflammation that occurs in humans during aging ${ }^{\mathbf{8}}$. The biological bases of inflamm-aging are still under investigation, but it is known that age-related inflammation is associated with the onset of common age-related diseases ${ }^{\mathbf{1 1}}$. The presence of circulating pro-inflammatory molecules (e.g. IL-6) but also of anti-inflammatory ones is not only a consequence, but also a causative phenomenon, and represents the result of ceaseless attrition of a variety of exogenous and endogenous pro-inflammatory agents, including nucleic acids ${ }^{11-13}$. The data available in Italy at March $24^{\text {th }}$ also convey that COVID-19 adverse outcome display gender-dependency. In particular, COVID19-lethality is substantially higher in men than in women $(70.9 \%$ of death occurs in males), (https://www.epicentro.iss.it/coronavirus/sars-cov-2-decessi-italia). In this regard, studies on the genetic basis of inflamm-aging showed that a gender-related genetic predisposition to produce high levels of IL-6 is found in aged men but not in women ${ }^{\mathbf{1 4}}$. Accordingly, the detrimental effect of inflamm-aging is postponed in women, who reach extreme limits of human lie-span with a higher chance $^{\mathbf{9}, 15}$. Therefore, the negative outcome of COVID-19 are more likely to occur in those people who show overt signs of inflamm-aging, i.e. elderly males affected by co-morbidity. Noteworthy, when SARS-CoV-1 infection was examined, an inadequate type-1 interferon-response, which represents the major viral clearance mechanism, was found ${ }^{7}$. At least in the early stages of viral infection, inflammation and type-1 anti-viral response are synergistic: antiviral response is aimed at promoting local mechanisms that prevent the spread of the viral agent, whilst inflammatory response promotes the local recruitment of inflammatory cells and the systemic spread. However, a reciprocal inhibitory effect between type-1 interferon anti-viral response and inflammation has been extensively reported ${ }^{\mathbf{7}, 16-26}$. Accordingly, as reported by the study on SARS-CoV-1 infected macaques, the type-1 interferon antiviral response is hampered in aged animals, which in fact develop a massive pro-inflammatory activation, but the administration of type-1 interferon reverses the unfavorable course of the infection ${ }^{7}$. Conceivably, in presence of inflamm-aging, SARS-CoV-2 response is likely to drift towards an uncontrollable local and systemic inflammation that may turn to be detrimental and potentially lethal. This setting is likely to turn out to be detrimental not only for the promotion of local and systemic inflammation but also for the inhibition of virus clearance in aged people $\mathbf{1 6}^{\mathbf{6}, 17}$. Following this reasoning, since women are endowed with enhanced type-1 interferon response compared to men the outcome of SARS-CoV-2 infection is expected to be more 
favorable in them ${ }^{27}$. Nevertheless, the adverse outcome of SARS-CoV-2 infection is not absent among young subjects. This finding points at the likelihood that (e.g. genetic) variability among individuals and/or ethnic groups may play a role in the SARS-CoV-2 disease outcome. In this commentary we will pinpoint the role of extracellular mtDNA and telomeric DNA sequences ${ }^{\mathbf{1 2}}$ as major actors in the interplay between of inflamm-aging and SARS-CoV-2 infection pathogenesis.

\section{1-Mitochondrial DNA: from a beneficial response to viral infections to a detrimental booster of systemic inflammation.}

Current data show that a single-strand positive RNA virus infections, similar to SARS-CoV2 viruses, i.e. Dengue virus, sets off a potent inflammatory response that involves the release of mtDNA outside the cell ${ }^{28}$. This phenomenon relies on passive and active (e.g. NET formation) or Netosis mechanisms ${ }^{\mathbf{2 9}}$ and may occur even via extracellular vesicles $(\mathrm{EVs})^{\mathbf{3 0 - 3 1}}$. Indeed, a large number of studies show that mtDNA is detectable in body fluids including plasma $\mathrm{EVs}^{\mathbf{3 0 , 3 2 , 3 3}}$. In turn, the release of mtDNA is an ancestral response mechanism to cell damage and it is nowadays regarded a major local and systemic trigger of inflammation ${ }^{\mathbf{3 4}}$. This phenomenon recalls the concept that mtDNA must be kept into the organelle until this latter is in a good shape and its functioning is preserved $^{34}$. Once damage has occurred, mtDNA leaks out into the cytoplasm and in the extracellular environment where it triggers inflammation, as well as the type-1 interferon antiviral response, by engaging cytoplasmic (AIM2) and/or endosomal/extracellular TLR9 detectors ${ }^{\mathbf{3 4 - 3 7}}$. Notably, it is worth noting that AIM2 promotes inflammation but tapers type-1 interferon antiviral response $^{\mathbf{1 6}}$. In line with these experimental evidence, current data indicate that mtDNA levels increase and correlate with the extent of damage, clinical evolution and the onset of multi-organ failure in patients affected by multiple systemic damage, septic conditions and Acute Respiratory Distress Syndrome ${ }^{36-40}$. Increased level of circulating MtDNA have been found in elderly people in presence/absence of systemic inflammation ${ }^{\mathbf{3 6 , 4 1}}$. On the basis of its ability to activate TLR9 ${ }^{36-38}$ and AIM2 ${ }^{35}$ circulating mtDNA molecule should be regarded as a powerful mediator of systemic inflammation and inflamm-aging. In this regard, studies on inflamm-aging and human longevity have shown that the a wide genetic variability of mtDNA genetic makeup among individuals and populations ( $\underline{\text { www.eupedia.com }}$ ) can be motivated not only by migrations and human history, but also by important functional differences among mtDNA variants ${ }^{\mathbf{4 2}}$. Since mitochondrial variants have different inflammatory and metabolic capacities they are likely to have an impact on systemic inflammation and/or sepsis still widely to be considered and explored ${ }^{\mathbf{4 3}}$ and some association between mtDNA genetic variability (haplogroups) and ICU outcomes have been reported ${ }^{\mathbf{4 3}, \mathbf{4 4}}$. Thus, studies on the level of mtDNA in plasma/EV of COVID-19 affected people in relationship with 
their age may help in understating whether the mtDNA-dependent systemic inflammation is deranged in aged people e/o in individuals (or populations) that, albeit at young ages, may be endowed with mtDNA variants that are peculiarly pro-inflammatory/and prone to be released.

\section{2-Telomeric shortening: the progressive decay of the anti-inflammatory back up that unleashes inflammation.}

Genomic (g)DNA that leaks out the nucleus cooperates with mtDNA in triggering the inflammatory response $^{\mathbf{1 2}}$. Interestingly, gDNA is not pro-inflammatory in all the experimental settings ${ }^{\mathbf{1 2}}$, this finding leads to propose that gDNA contains features (that may vary with cells of origin, age of the individual and epigenetic changes) that can affect its pro-inflammatory capability ${ }^{45}$. In particular, telomeric DNA fragments have been shown to have anti-inflammatory action on extracellular and cytoplasmic DNA receptors ${ }^{\mathbf{1 2} 46}$. Telomeres are double-stranded DNA sequences that protect the open ends of chromosomes ${ }^{47}$. The shortening of the telomeres with cellular aging has long been known for at least half a century ${ }^{\mathbf{4 8 , 4 9}}$. Notably, the length of the telomere decreases not only during cellular aging but also in cells from aged subjects affected by common age related diseases ${ }^{\mathbf{1 0 , 5 0 , 5 1}}$. Upon telomere shortening, a state of replicative arrest with consequent cellular senescence ensues, followed by the activation of inflammatory cytokines release (Senescence associated secretory phenotype, SASP) ${ }^{\mathbf{5 2 , 5 3}}$. Nowadays, SASP is considered as a major source of inflammaging ${ }^{\mathbf{1 1}}$. Telomere shortening also occurs when cells are subjected to a major stress such as viral infection, even in absence of any cell proliferation ${ }^{54}$. Different mechanisms of telomere shortening have been proposed $^{49}$. In particular, it has been observed that the telomere is actively kicked off the nucleus and can be found in the cytoplasm and in extracellular fluids, including plasma EVs ${ }^{\mathbf{1 2 , 5 5 , 5 6}}$. Therefore, telomere shortening can be also attributed to an active telomere fragments shedding which, as indicated above, may exert its intrinsic anti-inflammatory function as a free molecule ${ }^{\mathbf{4 6}}$. Indeed, the anti-inflammatory activity of telomeric repeats is mediated by the inhibition of innate immunity receptors such as TLR $-9^{47}$, AIM $2^{57}$. In this regard, the data indicate that the free telomeric DNA fragment can favorably switch off the mtDNA/TLR-9/NF-kappaB axis following ssRNA virus infection ${ }^{19}$. In addition, free telomeric DNA dampens the mtDNA/TLR-9/NF-kappaB axis in the respiratory system where the massive release of mtDNA following endotracheal intubation has been described ${ }^{37}$. Following these findings, the progressive loss of the telomeric anti-inflammatory reserve with aging is likely to be associated with the phenomenon of inflamm-aging ${ }^{10,12}$. In other words, gDNA released from cells during death or active phenomena ${ }^{29}$ (NET or netosis) may exert pro-or anti-inflammatory activity depending upon the amount of its telomeric content. In regard to 
this issue, it has been demonstrated that gDNA has a limited ability to induce inflammation through TLR-9, but that this activity is greatly increased after the cleavage of telomeric ends ${ }^{\mathbf{5 8}}$. Therefore, we expect that young subjects are endued with an abundant anti-inflammatory reserve that would, on average, worn off in the elderly, especially in those suffering from comorbidities associated with shortened telomeres ${ }^{\mathbf{5 0 5 1 , 5 9}}$. In case of an acute event, such as SARS-CoV-2 infection, the insufficient amount of telomere in extracellular DNA pool will unleash its pro-inflammatory potential that will add up to that of mtDNA. This scenario envisages also that young people, endued with short telomeres for environmental and/or genetic reasons will be more likely to undergo severe prognosis upon SARS-CoV-2 infection. The pictures may even more complex, by taking into account that the shortening of the telomeres triggers the transcription of an mRNA named as Telomere repeat-containing RNA (TERRA) ${ }^{\mathbf{6 0}}$. When TERRA binds to telomere ends it forms the so called DNA:RNA hybrids ${ }^{60}$. Notably, RNA:DNA hybrids, irrespective of their sequence, are powerful activators of inflammation via TLR-9 and cytoplasmic DNA sensors ${ }^{\mathbf{6 1 , 6 2}}$. These findings suggests that gDNA with shortened telomeres, not only is devoid of its intrinsic anti-inflammatory reservoir, but also contains increased levels of pro-inflammatory telomeric RNA:DNA hybrids. This scenario is more likely to occur in aged people and/or in those people who are affected by genetic/environmental causes of telomere shortening. Notably, EVs that contain TERRA and its cognate telomeric DNA sequence are likely to contain valuable amounts of RNA:DNA hybrids and have been demonstrated to exert a potent pro-inflammatory activity ${ }^{57}$. As plasma EVs contain TERRA, telomeric DNA and mtDNA ${ }^{\mathbf{3 0 , 5 6}}$ these nano-sized structures may exert a crucial role in controlling the pro/anti-inflammatory balancing of inflamm-aging as well as SARS-CoV-2 infection. Overall the above depicted scenery foresees that the assessment of telomeres DNA and TERRA RNA (as well as their cognate RNA:DNA hybrids) in the plasma (and in plasma EVs) would provide a useful tool to assess the DNA dependent inflammatory make-up of SARS-CoV-2 affected patients

\section{3-How modeling inflamm-aging may help in understanding COVID-19 pandemic.}

The interpretative model described above even accommodates the wide gender disparity of COVID19 lethality which amounts at least in Italy, to a male vs female ratio of $71,4 \%$ vs $29,6 \%$ (https://www.epicentro.iss.it/coronavirus/sars-cov-2-decessi-italia). Indeed, elderly man especially those affected by age related disease are endowed with shortened telomeres compared to aged matched women ${ }^{\mathbf{6 3}}$. Moreover, mtDNA instability is higher in males ${ }^{\mathbf{6 3}}$, thus, the pro-inflammatory unbalancing is more likely to occur in aged males which suffer from an accelerated inflamm-aging compared to females ${ }^{\mathbf{6 4}}$. Moreover, the model makes an attempt not only to explain the deleterious 
effects of SARS-CoV-2 in elderly patients, but also allows explaining the exceptions, i.e. those young people who undergo adverse outcomes and those very old patients who, unexpectedly, recover. In fact, it is known that our chronologic age does not exactly match to that on the identity card but we are characterized by a biological age ${ }^{65}$. Biological age can be measured throughout a variety of molecular parameters including the extent of epigenetic changes in certain sites of our genome ${ }^{66}$. Moreover, there are hints showing that some "unusual people, such as long living ones have an decelerated rate of biological aging ${ }^{67}$. Therefore, there are young people who are biologically older than expected and old ones that are biologically younger than expected ${ }^{\mathbf{4 5 , 6 7}}$ (extreme case are the centenarians). This reasoning is valid also for telomeres, whose length are unexpectedly long in centenarians $\mathbf{4 5}^{\mathbf{4 5}}$ corroborating the hypotesis that are exceptional individuals with a unique genetic and/or epigenetic background ${ }^{68}$. Consequently, based on the premises above described, the characterization of inflamm-aging and the mtDNA/telomere make-up of the patient may help the clinicians in the triage and/or in the follow up of SARS-CoV-2 affected people. In particular, determinations may be performed at a low cost and with very basic laboratory equipment: namely, assessing telomeric and mtDNA in plasma and PBMC to test the hypothesis that the lower is the mtDNA/Telomere ratio the worse is prognosis. The same assays in the plasma EVs fraction may be more informative, even though at present they may be prohibitive or ratelimiting for most laboratories. Also the assessment of mtDNA genetic variability by deep sequencing/site specific PCR assays will be suitable to test the hypothesis that mtDNA variants that are more/less common in certain ethnic/geographic groups differ in their pro/anti-inflammatory triggering potential. The pro-inflammatory capability of the patient DNA obtained from plasma (or plasma EVs) can be tested in vitro on target cells. This latter is a very specialized assay that requires experienced laboratories of cell biology. Nevertheless, the model above described not only proposes insight into the understanding of COVID-19 pathophysiology, but also paves the way to provide upgraded rationale for the repurposing of anti-inflammatory drugs alone/or in combination in COVID-19 disease. In this regard, global (as well as Italian) phase III clinical trial in which commercially available anti-IL-6 is administered to COVID-19 patients has been undertaken (NCT04317092; NCT04315480; https://www.aifa.gov.it/documents/20142). In turn, a global clinical trials proposed by WHO are aimed at testing anti-viral drugs (eg. Remdesivir, Lopinavir, NCT04280705, NCT04315948) alone or in combination with type I interferon (NCT04293887, NCT04320238) and/or chloroquine/hydroxychloroquine (NCT04303507, NCT04307693, NCT04318015). These drugs are well implemented in the model we have proposed. In particular, chloroquine may not act as generic "anti-inflammatory drug” but it may hampers SARS-CoV2 pathogenesis via its capability to halt TLR9 pathway during sepsis ${ }^{69}$. The above quoted repurposing 
armory may be enriched by additional anti-inflammatory drugs-based approaches. DNA oligonucleotides containing the telomeric sequence, though have not yet reached the use in humans but have been indeed explored in several in vitro and in vivo models, in which showed the ability to inhibit the TLR9 and AIM2-driven inflammatory pathways ${ }^{28,37,46,57}$. Nevertheless, telomeric repeats belong to a large family of polyguanosine rich (G-rich) DNA oligonucleotides that exert a variety of anti-inflammatory activities in various experimental settings ${ }^{\mathbf{7 0 - 7 3}}$. Intriguingly, Defibrotide, a drug made by a mixture of G-rich oligonucleotides is also endowed with a potent anti-inflammatory activity ${ }^{74}$. Currently, Defibrotide is employed in the treatment of Veno Occlusive Disease (VOD), a life-threatening complication due to liver endothelial injury causing embolization of erythrocytes, following hematopoietic stem cell transplantation ${ }^{74-76}$. Moreover, Defibrotide is currently tested in several inflammatory conditions such as the prevention of acute Graft versus host disease (GVHD) (NCT03339297) and neurotoxicity after CAR-T cell therapy (NCT03954106). In conclusion, the above-described scenario, summarized in figure 1, depicts how studies on inflamm-aging may help to understand and combat COVID-19 pandemic. Hopefully, this approach will help the ongoing struggling against the unprecedented pandemic that is spreading worldwide in 2020.

\section{Reference}

1. Callaway E, Cyranoski D, Mallapaty S, Stoye E, Tollefson J. The coronavirus pandemic in five powerful charts. Nature 2020; 579(7800):482-483.

2. Chen X, Zhao B, QU Y, Chen Y, ZXiong J et al. Detectable serum SARS-CoV-2 viral load (RNAaemia) is closely associated with drastically elevated interleukin 6 (IL-6) level in critically ill COVID-19 patients. medRxiv in press https://doi.org/10.1101/2020.02.29.20029520

3. Zhou F, Yu T, Du R, Fan G, Liu Y, et al. Clinical course and risk factors for mortality of adult inpatients with COVID-19 in Wuhan, China: a retrospective cohort study. Lancet 2020; pii: S0140-6736(20)30566-3. doi:

4. Mehta P, McAuley DF, Brown M, Sanchez E, Tattersall RS, Manson JJ. HLH Across Speciality Collaboration, UK. COVID-19: consider cytokine storm syndromes and immunosuppression. Lancet 2020; pii: S0140-6736(20)30628-0. doi:10.1016/S01406736(20)30628-0. [Epub ahead of print]

5. Ruan Q, Yang K, Wang W, Jiang L, Song J. Clinical Predictors of Mortality Due to COVID-19 Based on an Analysis of Data of 150 Patients From Wuhan, China. Intensive Care Med. 1-3 2020 
6. Remuzzi A, Remuzzi G. COVID-19 and Italy: what next? Lancet. 2020 Mar 13. pii:S01406736(20)30627-9.

7. Smits SL, de Lang A, van den Brand JM, Leijten LM, van IJcken WF, et al. Exacerbated innate host response to SARS-CoV in aged non-human primates. PLoS Pathog. 2010;6(2):e1000756.

8. Franceschi C, Bonafè M, Valensin S, Olivieri F, De Luca M, et al. Inflamm-aging. An evolutionary perspective on immunosenescence. Ann N Y Acad Sci. 2000; 908:244-54. Review.

9. Franceschi C, Garagnani P, Parini P, Giuliani C, Santoro A. Inflammaging: a new immunemetabolic viewpoint for age-related diseases. Nat Rev Endocrinol. 2018;14(10):576-590.

10. Bonafè M, Sabbatinelli J, Olivieri F. Exploiting the telomere machinery to put the brakes on inflamm-aging. Ageing Res Rev. 2020; 59:101027.

11. Franceschi C, Campisi J. Chronic inflammation (inflammaging) and its potential contribution to age-associated diseases. J Gerontol A Biol Sci Med Sci. 2014; Suppl 1:S4-9.

12. Storci G, De Carolis S, Olivieri F, Bonafè M. Changes in the biochemical taste of cytoplasmic and cell-free DNA are major fuels for inflamm-aging. Semin Immunol. 2018; 40:6-16.

13. Morrisette V, Cohen AA, Fülöp T, Riesco É, Legault V, et al. Inflamm-aging does not simply reflect increases in pro-inflammatory markers. Mech Ageing Dev. 2014; 139:49-57.

14. Bonafè M, Olivieri F, Cavallone L, Giovagnetti S, Mayegiani F, et al. A gender--dependent genetic predisposition to produce high levels of IL-6 is detrimental for longevity. Eur J Immunol. 2001;31(8):2357-61.

15. Albani D, Mazzuco S, Polito L, Batelli S, Biella G, et al. Insulin-like growth factor 1 receptor polymorphism rs2229765 and circulating interleukin-6 level affect male longevity in a population-based prospective study (Treviso Longeva--TRELONG). Aging Male. 2011; 14(4):257-64.

16. Banerjee I, Kailasan Vanaja S, Kate A, Rathinam V. Control of type I interferon responses by the AIM2 inflammasome. J Immunol May 1, 2017, 198 (1 Supplement) 64.3;

17. Menachery VD, Eisfeld AJ, Schäfer A, Josset L, Sims AC et al. Pathogenic influenza viruses and coronaviruses utilize similar and contrasting approaches to control interferonstimulated gene responses. mBio. 2014;5(3):e01174-14.

18. Chan RW, Chan MC, Agnihothram S, Chan LL, Kuok DI, et al. Tropism of and innate immune responses to the novel human betacoronavirus lineage $\mathrm{C}$ virus in human ex vivo respiratory organ cultures. J Virol. 2013; 87(12):6604-14. 
19. Kindler E, Thiel V. To sense or not to sense viral RNA--essentials of coronavirus innate immune evasion. Curr Opin Microbiol. 2014; 20:69-75.

20. Lau SKP, Lau CCY, Chan KH, Li CPY, Chen H. Delayed induction of proinflammatory cytokines and suppression of innate antiviral response by the novel Middle East respiratory syndrome coronavirus: implications for pathogenesis and treatment. J Gen Virol. 2013; 94(Pt 12):2679-2690.

21. Zielecki F, Weber M, Eickmann M, Spiegelberg L, Zaki AM, et al. Human cell tropism and innate immune system interactions of human respiratory coronavirus EMC compared to those of severe acute respiratory syndrome coronavirus. J Virol. 2013; 87(9):5300-4.

22. Aman MJ, Tretter T, Eisenbeis I, Bug G, Decker T, et al. Interferon-alpha stimulates production of interleukin-10 in activated CD4+ T cells and monocytes. Blood. 1996; 87(11):4731-6.

23. Ganster RW, Guo Z, Shao L, Geller DA. Differential effects of TNF-alpha and IFN-gamma on gene transcription mediated by NF-kappaB-Stat1 interactions. J Interferon Cytokine Res. 2005; 25(11):707-19.

24. Pauli EK, Schmolke M, Wolff T, Viemann D, Roth J, et al. Influenza A virus inhibits type I IFN signaling via NF-kappaB-dependent induction of SOCS-3 expression. PLoS Pathog. 2008; 4(11):e1000196.

25. Wei L, Sandbulte MR, Thomas PG, Webby RJ, Homayouni R, Pfeffer LM. NFkappaB negatively regulates interferon-induced gene expression and anti-influenza activity. J Biol Chem. 2006; 281(17):11678-84.

26. Nagata N, Iwata N, Hasegawa H, Fukushi S, Harashima A, et al. Mouse-passaged severe acute respiratory syndrome-associated coronavirus leads to lethal pulmonary edema and diffuse alveolar damage in adult but not young mice. Am J Pathol. 2008; 172(6):1625-37.

27. Klein SL, Flanagan KL. Sex differences in immune responses. Nat Rev Immunol. 2016;16(10):626-38.

28. Lai JH, Wang MY, Huang CY, Wu CH, Hung LF, et al . Infection with the dengue RNA virus activates TLR9 signaling in human dendritic cells. EMBO Rep. 2018;19(8). pii: e46182.

29. Schönrich G, Raftery MJ. Neutrophil Extracellular Traps Go Viral. Front Immunol. 2016; 7:366. eCollection 2016. Review.

30. Guescini M, Guidolin D, Vallorani L, Casadei L, Gioacchini AM, et al. C2C12 myoblasts release micro-vesicles containing mtDNA and proteins involved in signal transduction. Exp Cell Res. 2010;316(12):1977-84. 
31. Sansone P, Savini C, Kurelac I, Chang Q, Amato LB, et al. Packaging and transfer of mitochondrial DNA via exosomes regulate escape from dormancy in hormonal therapyresistant breast cancer. Proc Natl Acad Sci U S A. 2017;114(43):E9066-E9075.

32. Meddeb R, Dache ZAA, Thezenas S, Otandault A, Tanos R, et al. Quantifying circulating cell-free DNA in humans. Sci Rep. 2019;9(1):5220.

33. Thurairajah K, Briggs GD, Balogh ZJ. The source of cell-free mitochondrial DNA in trauma and potential therapeutic strategies. Eur J Trauma Emerg Surg. 2018 ;44(3):325-334

34. Zhang Q, Raoof M, Chen Y, Sumi Y, Sursal T, et al. Circulating mitochondrial DAMPs cause inflammatory responses to injury. Nature. 2010;464(7285):104-7.

35. Bae JH, Jo SI, Kim SJ, Lee JM, Jeong JH, et al. Circulating Cell-Free mtDNA Contributes to AIM2 Inflammasome-Mediated Chronic Inflammation in Patients with Type 2 Diabetes. Cells. 2019;8(4).

36. Itagaki K, Kaczmarek E, Lee YT, Tang IT, Isal B, et al. Mitochondrial DNA released by trauma induces neutrophil extracellular traps. PLoS One. 2015;10(3):e0120549.

37. Puyo CA, Earhart A, Staten N, Prince OA, Haug C, et al. Endotracheal intubation results in acute tracheal damage induced by mtDNA/TLR9/NF-кB activity. J Leukoc Biol. 2019; 105(3):577-587.

38. Simmons JD, Lee YL, Mulekar S, Kuck JL, Brevard SB, et al. Elevated levels of plasma mitochondrial DNA DAMPs are linked to clinical outcome in severely injured human subjects. Ann Surg. 2013; 258(4):591-6; discussion 596-8.

39. Nakahira K, Kyung SY, Rogers AJ, Gazourian L, Youn S, et al. Circulating mitochondrial DNA in patients in the ICU as a marker of mortality: derivation and validation. PLoS Med. 2013; 10(12):e1001577;

40. Faust HE, Reilly JP, Anderson BJ, Ittner CAG, Forker CM, et al. Plasma Mitochondrial DNA Levels Are Associated With ARDS in Trauma and Sepsis Patients. Chest. 2020;157(1):67-76.

41. Pinti M, Cevenini E, Nasi M, De Biasi S, Salvioli S, et al. Circulating mitochondrial DNA increases with age and is a familiar trait: Implications for "inflamm-aging". Eur J Immunol. 2014; 44(5):1552-62.

42. Chen A, Raule N, Chomyn A, Attardi G. Decreased reactive oxygen species production in cells with mitochondrial haplogroups associated with longevity. PLoS One. 2012;7(10):e46473. 
43. Lorente L, Iceta R, Martín MM, López-Gallardo E, Solé-Violán J, et al. Survival and mitochondrial function in septic patients according to mitochondrial DNA haplogroup. Crit Care. 2012;16(1):R10.

44. Jiménez-Sousa MA, Tamayo E, Guzmán-Fulgencio M, Heredia M, Fernández-Rodríguez A, et al. Mitochondrial DNA haplogroups are associated with severe sepsis and mortality in patients who underwent major surgery. J Infect. 2015;70(1):20-9.

45. Storci G, De Carolis S, Papi A, Bacalini MG, Gensous N, et al. Genomic stability, antiinflammatory phenotype, and up-regulation of the RNAseH2 in cells from centenarians. Cell Death Differ. 2019; 26(9):1845-1858.

46. Gursel I, Gursel M, Yamada H, Ishii KJ, Takeshita F, Klinman DM. Repetitive elements in mammalian telomeres suppress bacterial DNA-induced immune activation. J Immunol. 2003; 171(3):1393-400.

47. Palm W, de Lange T. How shelterin protects mammalian telomeres. Annu Rev Genet. 2008; 42:301-34.

48. HAYFLICK L, MOORHEAD PS. The serial cultivation of human diploid cell strains. Exp Cell Res. 1961; 25:585-621.

49. Aguado J, Sola-Carvajal A, Cancila V, Revêchon G, Ong PF, et al. Inhibition of DNA damage response at telomeres improves the detrimental phenotypes of Hutchinson-Gilford Progeria Syndrome. Nat Commun. 2019; 10(1):4990.

50. Campisi J. Cellular senescence as a tumor-suppressor mechanism. Trends Cell Biol. 2001;11(11):S27-31.

51. Rodier F, Coppé JP, Patil CK, Hoeijmakers WA, Muñoz DP, et al. Persistent DNA damage signalling triggers senescence-associated inflammatory cytokine secretion. Nat Cell Biol. 2009; 11(8):973-9.

52. Armanios M. Telomeres and age-related disease: how telomere biology informs clinical paradigms. J Clin Invest. 2013; 123(3):996-1002.

53. Herrmann M, Pusceddu I, März W, Herrmann W. Telomere biology and age-related diseases. Clin Chem Lab Med. 2018; 56(8):1210-1222.

54. Wang Z, Deng Z, Tutton S, Lieberman PM. The Telomeric Response to Viral Infection. Viruses. 2017; 9;9(8):218.

55. Byrd AK, Zybailov BL, Maddukuri L, Gao J, Marecki JC, et al. Evidence That Gquadruplex DNA Accumulates in the Cytoplasm and Participates in Stress Granule Assembly in Response to Oxidative Stress. J Biol Chem. 2016;291(34):18041-57. 
56. Wang Z, Deng Z, Dahmane N, Tsai K, Wang P, et al. Telomeric repeat-containing RNA (TERRA) constitutes a nucleoprotein component of extracellular inflammatory exosomes. Proc Natl Acad Sci U S A. 2015;112(46):E6293-300.

57. Kaminski JJ, Schattgen SA, Tzeng TC, Bode C, Klinman DM, Fitzgerald KA. Synthetic oligodeoxynucleotides containing suppressive TTAGGG motifs inhibit AIM2 inflammasome activation. J Immunol. 2013;191(7):3876-83.

58. Goldfarb IT, Adeli S, Berk T, Phillippe M. Fetal and Placental DNA Stimulation of TLR9: A Mechanism Possibly Contributing to the Pro-inflammatory Events During Parturition. Reprod Sci. 2018 May;25(5):788-796.

59. Zhao J, Miao K, Wang H, Ding H, Wang DW. Association between telomere length and type 2 diabetes mellitus: a meta-analysis. PLoS One. 2013; 8(11):e79993.

60. Balk B, Maicher A, Dees M, Klermund J, Luke-Glaser S, Bender K, et al. Telomeric RNADNA hybrids affect telomere-length dynamics and senescence. Nat Struct Mol Biol. 2013; 20(10):1199-205.

61. Rigby RE, Webb LM, Mackenzie KJ, Li Y, Leitch A, et al. RNA:DNA hybrids are a novel molecular pattern sensed by TLR9. EMBO J. 2014; 33(6):542-58.

62. Mankan AK, Schmidt T, Chauhan D, Goldeck M, Höning K, et al. Cytosolic RNA:DNA hybrids activate the cGAS-STING axis. EMBO J. 2014; 33(24):2937-46.

63. Fischer KE, Riddle NC. Sex Differences in Aging: Genomic Instability. J Gerontol A Biol Sci Med Sci. 2018; 16;73(2):166-174.

64. Márquez EJ, Chung CH, Marches R, Rossi RJ, Nehar-Belaid D, et al. Sexual-dimorphism in human immune system aging. Nat Commun. 2020;11(1):751.

65. Horvath S. DNA methylation age of human tissues and cell types. Genome Biol. 2013;14(10):R115.

66. Gensous N, Bacalini MG, Pirazzini C, Marasco E, Giuliani C, et al. The epigenetic landscape of age-related diseases: the geroscience perspective. Biogerontology. 2017; 18(4):549-559.

67. Horvath S, Pirazzini C, Bacalini MG, Gentilini D, Di Blasio AM, et al. Decreased epigenetic age of PBMCs from Italian semi-supercentenarians and their offspring. Aging (Albany NY). 2015; 7(12):1159-70.

68. Franceschi C, Bonafè M. Centenarians as a model for healthy aging. Biochem Soc Trans. 2003; 31(2): 457-61. 
69. Yasuda H, Leelahavanichkul A, Tsunoda S, Dear JW, Takahashi Y, et al. Chloroquine and inhibition of Toll-like receptor 9 protect from sepsis-induced acute kidney injury. Am $\mathbf{J}$ Physiol Renal Physiol. 2008; 294(5): F1050-8.

70. Bayik D, Gursel I, Klinman DM. Structure, mechanism and therapeutic utility of immunosuppressive oligonucleotides. Pharmacol Res. 2016; 105:216-25.

71. Ohto U, Shibata T, Tanji H, Ishida H, Krayukhina E, et al. Structural basis of CpG and inhibitory DNA recognition by Toll-like receptor 9. Nature. 2015;520(7549):702-5.

72. Römmler F, Hammel M, Waldhuber A, Müller T, Jurk M, et al. Guanine-modified inhibitory oligonucleotides efficiently impair TLR7- and TLR9-mediated immune responses of human immune cells. PLoS One. 2015;10(2):e0116703.

73. Lenert PS. Classification, mechanisms of action, and therapeutic applications of inhibitory oligonucleotides for Toll-like receptors (TLR) 7 and 9. Mediators Inflamm. 2010; 2010:986596.

74. Pescador R, Capuzzi L, Mantovani M, Fulgenzi A, Ferrero ME. Defibrotide: properties and clinical use of an old/new drug. Vascul Pharmacol. 2013; 59(1-2):1-10.

75. Palomo M, Mir E, Rovira M, Escolar G, Carreras E, Diaz-Ricart M. What is going on between defibrotide and endothelial cells? Snapshots reveal the hot spots of their romance. Blood. 2016; 127(13):1719-27.

76. Richardson PG, Carreras E, Iacobelli M, Nejadnik B. The use of defibrotide in blood and marrow transplantation. Blood Adv. 2018; 2(12):1495-1509. 